\title{
THE IDEAL STRUCTURE OF IDEMPOTENT-GENERATED TRANSFORMATION SEMIGROUPS
}

\author{
by M. A. REYNOLDS and R. P. SULLIVAN
}

(Received 29th August 1984)

\section{Introduction}

Let $X$ be a set and $\mathscr{T}_{X}$ the semigroup (under composition) of all total transformations from $X$ into itself. In ([6], Theorem 3) Howie characterised those elements of $\mathscr{T}_{X}$ that can be written as a product of idempotents in $\mathscr{T}_{X}$ different from the identity. We gather from review articles that his work was later extended by Evseev and Podran $[3,4]$ (and independently for finite $X$ by Sullivan [15]) to the semigroup $\mathscr{P}_{X}$ of all partial transformations of $X$ into itself. Howie's result was generalized in a different direction by Kim [8], and it has also been considered in both a topological and a totally ordered setting (see [11] and [14] for brief summaries of this latter work). In addition, Magill [10] investigated the corresponding idea for endomorphisms of a Boolean ring, while J. A. Erdos [2] resolved the analogous problem for linear transformations of a finite-dimensional vector space.

In this paper we return to Howie's original article and first determine the ideals of the semigroup $\mathscr{E}_{X}$ generated by the idempotents in $\mathscr{T}_{X}$ different from the identity. Next we characterise Green's relations on $\mathscr{E}_{X}$ and use our result to produce a new class of 0bisimple regular semigroups. Finally we consider the extension of our work to the partial case.

\section{Ideal structure}

Throughout this paper we shall in the main use the notation of [1] but occasionally abbreviate it for the purpose of convenience. In particular, if $\alpha \in \mathscr{T}_{X}$ we write $r(\alpha)=|X \alpha|$ and can call this the rank of $\alpha$.

Howie showed in ([6], Theorem 1) that if $X$ is finite then $\mathscr{E}_{X}=\left\{\alpha \in \mathscr{T}_{X}: r(\alpha)<|X|\right\}$. Since the ideals of this semigroup are well-known (cf. [1], Vol. 2, Theorem 10.59) we assume for the remainder of this section that $|X|=\kappa \geqq \aleph_{0}$. In [6] Howie described the elements of $\mathscr{E}_{X}$ in this case via three concepts: if $\alpha \in \mathscr{T}_{X}$ we put

$$
\begin{array}{ll}
D(\alpha)=X \backslash X \alpha & \text { and } d(\alpha)=|D(\alpha)| \\
S(\alpha)=\{x \in X: x \alpha \neq x\} & \text { and } s(\alpha)=|S(\alpha)| \\
C(\alpha)=\cup\left\{t \alpha^{-1}:\left|t \alpha^{-1}\right| \geqq 2\right\} & \text { and } c(\alpha)=|C(\alpha)|
\end{array}
$$


and we refer to the cardinals $d(\alpha), s(\alpha)$ and $c(\alpha)$ as the defect, shift and collapse of $\alpha$, respectively. Howie proved in ([6], Theorem 3) that $\mathscr{E}_{X}$ is the disjoint union of two semigroups:

$$
\begin{aligned}
& V=\left\{\alpha \in \mathscr{T}_{X}: s(\alpha)<\aleph_{0} \text { and } d(\alpha) \neq 0\right\} \\
& H=\left\{\alpha \in \mathscr{T}_{X}: s(\alpha)=d(\alpha)=c(\alpha) \geqq \aleph_{0}\right\} .
\end{aligned}
$$

That $V$ is in fact a semigroup follows from ([6], Lemmas 2 and 5), and that $H$ is a semigroup follows from ([6], Lemmas 6 and 7). Since we will need to refer to the last of these Lemmas quite often, we re-state it here for convenience (and note in passing that the original proof contained a significant error that was neatly corrected in [7]).

Lemma 1. If $\alpha \in H, \beta \in \mathscr{T}_{X}$ and $s(\beta)<s(\alpha)$ then both $\alpha \beta$ and $\beta \alpha$ have shift, defect and collapse equal to that of $\alpha$.

In this section we aim to describe the ideals $I$ of $\mathscr{E}_{X}$ : note that $I=(I \cap V) \cup(I \cap H)$ and if $I \cap V$ and $I \cap H$ are non-empty they are ideals of $V$ and $H$ respectively; hence our first task will be to determine the ideals of $V$ and of $H$. It seems that Vorobev [18] has described the ideals of a semigroup closely allied to $V$ : namely, the set of all $\alpha \in \mathscr{T}_{X}$ with $s(\alpha)<\aleph_{0}$ ([17] may also be relevant: it is listed in [9] but has not been reviewed and was unavailable to us). For completeness we provide a proof of the following.

Theorem 1. Let $n \in \mathbb{Z}^{+}$and $V_{n}=\{\alpha \in V: d(\alpha) \geqq n\}$. Then $V_{n}$ is an ideal of $V$ and every ideal of $V$ equals some $V_{n}$. Moreover, each $V_{n}$ is principal and generated by an element with defect $\boldsymbol{n}$.

Proof. Let $\alpha \in V_{n}$ and $\beta \in V$. Since $D(\alpha) \subseteq D(\beta \alpha)$, we have $\beta \alpha \in V_{n}$. Although after some reflection it is intuitively clear that also $\alpha \beta \in V_{n}$, a convincing argument is somewhat longer. Firstly we assert that

$$
[S(\beta) \cup D(\alpha)] \cap X \alpha \beta \subseteq[S(\beta) \backslash D(\alpha)] \beta .
$$

For, if $x \in S(\beta) \cup D(\alpha)$ and $x=y \alpha \beta$ for some $y \in X$ then $y \alpha \in S(\beta)$ : otherwise, $x=(y \alpha) \beta=$ $y \alpha \notin S(\beta)$ implies $x=y \alpha \in D(\alpha)$, a contradiction. Hence $y \alpha \in S(\beta) \backslash D(\alpha)$, and our assertion follows. Now we put $Y=S(\beta) \cup D(\alpha)$ and note that

$$
|Y \cap X \alpha \beta| \leqq|S(\beta) \backslash D(\alpha)|
$$

where

$$
|Y|=|Y \cap X \alpha \beta|+|Y \cap D(\alpha \beta)|
$$

Hence we have

$$
d(\alpha \beta) \geqq|Y \cap D(\alpha \beta)|=|Y|-|Y \cap X \alpha \beta| \geqq|Y|-|S(\beta) \backslash D(\alpha)|=d(\alpha) \geqq n .
$$


For the converse we assume $I$ is an ideal of $V$, choose $\alpha \in I$ with minimal defect, and put $d(\alpha)=n$. Then $I \subseteq V_{n}$. Let $\beta \in V_{n}$ and put $Z=E(\alpha) \cup E(\beta)$, where $E(\gamma)$ denotes $S(\gamma) \cup S(\gamma) \gamma$ for each $\gamma \in V$. Note that both $\alpha$ and $\beta$ map $Z$ into itself, and fix $X \backslash Z$ pointwise. Hence $D(\alpha) \cup D(\beta) \subseteq Z$. Put $\alpha_{1}=\alpha \mid Z$ and $\beta_{1}=\beta \mid Z$, and note that since $\alpha_{1}$ fixes $E(\beta) \backslash E(\alpha)$, we have $d\left(\alpha_{1}\right)=d(\alpha)$. Likewise $d\left(\beta_{1}\right)=d(\beta)$ and, since

$$
r\left(\alpha_{1}\right)+d\left(\alpha_{1}\right)=r\left(\beta_{1}\right)+d\left(\beta_{1}\right)=|Z|<\aleph_{0}
$$

where $d\left(\beta_{1}\right) \geqq d\left(\alpha_{1}\right) \neq 0$, we conclude that $r\left(\beta_{1}\right) \leqq r\left(\alpha_{1}\right)<|Z|$. At this point we can invoke the well-known characterisation of Green's $\mathscr{J}$-relation on $\mathscr{T}_{Z}$ (cf. [1], Vol. 1, pp. 52-53) to assert that $\beta_{1}=\lambda_{1} \alpha_{1} \mu_{1}$ for some $\lambda_{1}, \mu_{1} \in \mathscr{T}_{Z}$. In fact, since $r\left(\alpha_{1}\right)<|Z|$, we can ensure that $d\left(\lambda_{1}\right)$ and $d\left(\mu_{1}\right)$ are both non-zero. So, by extending $\lambda_{1}$ and $\mu_{1}$ to the whole of $X$ in an obvious way, we obtain $\beta=\lambda \alpha \mu$ for some $\lambda, \mu \in V$; that is, $\beta \in I$ and we have shown $I=V_{n}$. That $V_{n}$ is a principal ideal is clear from the foregoing discussion.

According to ([1], Vol. 2, Theorem 10.59) the ideals of $\mathscr{T}_{X}$ take the form $I_{\xi}$ where for $1<\xi \leqq \kappa^{\prime}$

$$
I_{\xi}=\left\{\alpha \in \mathscr{T}_{X}: r(\alpha)<\xi\right\}
$$

If $\xi$ is finite then the Rees quotient semigroup $I_{\xi+1} / I_{\xi}$ is completely 0 -simple ([1], Vol. 2, Lemma 10.54). We assert that this is also true for the semigroups $V_{n} / V_{n+1}$ where $1 \leqq n<\aleph_{0} \leqq \kappa$. To show this we again use the set $E(\alpha)=S(\alpha) \cup S(\alpha) \alpha$ (called by Symons [16] the essential domain of $\alpha \in \mathscr{T}_{X}$ ); namely, if $\alpha, \beta \in V$ with $d(\alpha)=d(\beta)=n$, we put $Y=E(\alpha) \cup E(\beta)$ and observe that $\alpha_{1}=\alpha \mid Y$ and $\beta_{1}=\beta \mid Y$ are elements of $\mathscr{T}_{Y}$ with $r\left(\alpha_{1}\right)=r\left(\beta_{1}\right)<|Y|$. We can now follow the proof of ([1], Vol. 2, Lemma 10.54) to eventually conclude that $V_{n} / V_{n+1}$ is 0 -simple. Clearly, $V_{n} \backslash V_{n+1}$ contains idempotents. To show each of these is primitive, we again put $Y=E(\alpha) \cup E(\beta)$ where $\alpha, \beta$ are idempotents in $V$ with $\alpha \beta=\beta \alpha=\alpha$ and $d(\alpha)=d(\beta)$. Then $\alpha_{1} \beta_{1}=\beta_{1} \alpha_{1}=\alpha_{1}$ for idempotents $\alpha_{1}, \beta_{1} \in \mathscr{T}_{Y}$ with $r\left(\alpha_{1}\right)=r\left(\beta_{1}\right)<\mathcal{N}_{0}$. An argument similar to that in the reference already cited eventually leads us to $\alpha=\beta$. We have therefore shown

Theorem 2. If $1 \leqq n<\aleph_{0}$ then $V_{n} / V_{n+1}$ is a completely 0 -simple semigroup.

We assert that $V_{n} / V_{n+1}$ is not isomorphic to any $I_{m+1} / I_{m}$ with $m$ finite $\ldots$ simply because the cardinal of the first is $\kappa$ while that of the second is $2^{\kappa}$. To see this, recall that the set $\mathscr{F}$ of all finite subsets of $X$ has cardinal $\kappa([12]$, Theorem 22.17). If $\mathscr{F}$ is any finite subset of $X$ such that $|F|=n+1 \geqq 2$ then $V_{n} / V_{n+1}$ contains an idempotent that is constant on $F$ and fixes $X \backslash F$; hence, if $\left|V_{n} / V_{n+1}\right|=\varepsilon$ then $\varepsilon \geqq \kappa$. Now, to each $\alpha \in V_{n} \backslash V_{n+1}$ we can associate in a one-to-one fashion the element $\alpha \mid \bar{E}(\alpha)$ of $\mathscr{T}_{E(\alpha)}$. Hence if $m_{F}$ denotes the (finite) cardinal of $\mathscr{T}_{F}$ for each $F \in \mathscr{F}$ then $\varepsilon \leqq \Sigma m_{F}=\kappa$. On the other hand, if $F \in \mathscr{F}$ and $|F|=m$ then $I_{m+1} / I_{m}$ contains all maps from $X$ onto $F$ and there are $2^{\kappa}$ such maps (since there are $\left(2^{\kappa}\right)^{m}=2^{\kappa}$ ways of partitioning $X$ into a family of $m$ subsets of $X$ : cf. [12], Exercise 22.20). However the cardinal of $\mathscr{T}_{X}$ is $2^{\kappa}$ and so we have $\left|I_{m+1} / I_{m}\right|=2^{x}$.

It will become apparent after we have determined Green's $\mathscr{H}$-relation on $V$ in Section 3 that $V_{n} / V_{n+1}$ and $I_{m+1} / I_{m}$ are non-isomorphic for a less trivial reason: namely, the 
non-zero group $\mathscr{H}$-classes of $I_{m+1} / I_{m}$ are all isomorphic to the symmetric group $\mathscr{G}_{m}$ on $m$ letters (as observed in [1], Vol. 2, p. 226) whereas those of $V_{n} / V_{n+1}$ are all isomorphic to the group $\mathscr{G}\left(\kappa, \aleph_{0}\right)$ of all permutations of $\kappa$ letters with finite shift (as can be readily checked by mimicking the proof of Theorem 2.10(ii) in [1], Vol. 1).

We now turn to the problem of describing the ideal of $H$ : it happens that, just as in $V$, they form a chain, even though two cardinals are required for their description. To show this, we let

$$
H(\delta, \xi)=\{\alpha \in H: d(\alpha) \geqq \delta \quad \text { and } \quad r(\alpha)<\xi\}
$$

where $\aleph_{0} \leqq \delta \leqq \kappa$ and $2 \leqq \xi \leqq \kappa^{\prime}$.

Lemma 2. Each $H(\delta, \xi)$ is an ideal of $H$ and the set of all such distinct ideals forms a chain:

$$
H(\kappa, 2) \subseteq \cdots \subseteq H(\kappa, \xi) \subseteq \cdots \subseteq H\left(\kappa, \kappa^{\prime}\right) \subseteq \cdots \subseteq H\left(\aleph_{1}, \kappa^{\prime}\right) \subseteq H\left(\aleph_{0}, \kappa^{\prime}\right)
$$

Proof. Let $\alpha \in H(\delta, \xi)$ and $\beta \in H$, and suppose $s(\alpha)=d(\alpha)=c(\alpha)=a$ and $s(\beta)=d(\beta)=$ $c(\beta)=b$. If $b \leqq a$ then ([6], Lemmas 6 and 7) imply that both $\alpha \beta$ and $\beta \alpha$ have defect equal to $a(\geqq \delta)$. If $a>b$ then Lemma 1 above implies that both $\alpha \beta$ and $\beta \alpha$ have defect equal to $b>a \geqq \delta$. Since $r(\alpha \beta) \leqq \min \{r(\alpha), r(\beta)\}$ it therefore follows that $\alpha \beta, \beta \alpha \in H(\delta, \xi)$ and $H(\delta, \xi)$ is an ideal of $H$.

Now consider an arbitrary $H(\delta, \xi)$. If $\delta=\kappa$ (and $2 \leqq \xi \leqq \kappa^{\prime}$ ) we have an ideal in the first portion of the above chain, and if $\xi=\kappa^{\prime}$ (and $\left.\kappa_{0} \leqq \delta \leqq \kappa\right)$ we are in the second portion of the chain. On the other hand, since $|X|=\kappa \geqq \aleph_{0}$ and $X=X \alpha \cup(X \backslash X \alpha)$ for each $\alpha \in H$, we must have $d(\alpha)=\kappa$ if $r(\alpha)<\xi \leqq \kappa$; that is if $\delta<\kappa, \xi \leqq \kappa$ and $\alpha \in H(\delta, \xi)$ then $\alpha \in H(\kappa, \xi)$. Since $H(\kappa, \xi) \subseteq H(\delta, \xi)$, we deduce that $H(\delta, \xi)=H(\kappa, \xi)$ when $\delta<\kappa$ and $\zeta \leqq \kappa$.

Following ([1], Vol. 2, p. 241), for each $\alpha \in \mathscr{T}_{X}$, we write

$$
\alpha=\left(\begin{array}{c}
C_{m} \\
x_{m}
\end{array}\right)
$$

where $X \alpha=\left\{x_{m}: m \in M\right\}$ for some index set $M$ and $C_{m}=x_{m} \alpha^{-1}$ for each $m \in M$. To abbreviate notation, we adopt the convention (as in the reference just cited) of writing $\left\{x_{m}\right\}$ for $\left\{x_{m}: m \in M\right\}$, taking the subscript $m$ to signify the index set $M$ within a specific context.

Theorem 3. Every ideal of $H$ has the form $H(\delta, \xi)$ for some $\delta$, $\xi$. In particular, the principal ideals of $H$ are $H\left(\kappa, \eta^{\prime}\right)$ and $H\left(\varepsilon, \kappa^{\prime}\right)$ for some $\eta, \varepsilon$ satisfying $1 \leqq \eta \leqq \kappa$ and $\aleph_{0} \leqq \varepsilon \leqq \kappa$.

Proof. Suppose $I$ is an ideal of $H$. Let $\delta$ be the defect of an element of $I$ with minimal defect and let $\xi$ be the least cardinal greater than the ranks of all the elements of $I$. We assert that $I=H(\delta, \xi)$. Since $I \subseteq H(\delta, \xi)$, we therefore proceed to show that if $\beta \in H(\delta, \xi)$ then there exist $\alpha \in I$ and $\lambda, \mu \in H$ such that $\beta=\lambda \alpha \mu$. So, let $\beta \in H(\delta, \xi)$ and note 
that $r(\alpha)<r(\beta)<\xi$ for all $\alpha \in I$ contradicts the choice of $\xi$. Hence there exists $\alpha \in I$ with $r(\beta) \leqq r(\alpha)$. Put

$$
\beta=\left(\begin{array}{c}
B_{m} \\
x_{m}
\end{array}\right) \text { and } \alpha=\left(\begin{array}{c}
A_{n} \\
y_{n}
\end{array}\right)
$$

and choose a cross-section $\left\{a_{n}\right\}$ of $\left\{A_{n}\right\}$. Write $\left\{a_{n}\right\}=\left\{a_{m}\right\} \dot{\cup}\left\{a_{s}\right\}$, which is possible since $r(\alpha) \geqq r(\beta)$, and put

$$
\lambda=\left(\begin{array}{l}
B_{m} \\
a_{m}
\end{array}\right)
$$

Now suppose $d(\alpha)=d(\beta)=\kappa$. Since $\beta \in H$, we have $c(\beta)=\kappa$ and this means $c(\lambda)=s(\lambda)=\kappa$ (using [6], Lemma 3). If $r(\beta)<\kappa$ then $\left|X \backslash\left\{a_{m}\right\}\right|=\kappa$ and so $d(\lambda)=\kappa$; that is, $\lambda \in H$. If on the other hand $r(\beta)=\kappa$ then $r(\alpha)=\kappa$ and we can ensure that $|S|=\kappa$; that is, $\left\{a_{s}\right\} \subseteq D(\lambda)$ and again $\lambda \in H$. To define $\mu$, put $C=X \backslash\left\{y_{m}\right\}$ and note that $D(\alpha) \subseteq C$. Hence if we choose $z \in C$ and define

$$
\mu=\left(\begin{array}{ll}
y_{m} & C \\
x_{m} & z
\end{array}\right)
$$

then $c(\mu)=s(\mu)=\kappa$. In addition, $D(\beta) \backslash z \subseteq D(\mu)$ and so $d(\mu)=\kappa$. That is, $\mu \in H$ and we have $\beta=\lambda \alpha \mu$ as required.

Before considering the next case, note that if $r(\beta) \leqq r(\alpha)<\kappa$ then $d(\alpha)=d(\beta)=\kappa$ as above. Hence we may suppose $r(\alpha)=\kappa$. Suppose further that $d(\beta)=\kappa$. In this case, with the same notation as before, we immediately have $c(\lambda)=s(\lambda)=\kappa$. Moreover, since $r(\alpha)=\kappa$ we can ensure that $|S|=\kappa$. Then $d(\lambda)=\kappa$ and, since $\left\{y_{s}\right\} \subseteq C$, we also have $c(\mu)=s(\mu)=\kappa$ together with $d(\mu)=\kappa$ (as before).

Hence we may now assume $r(\alpha)=\kappa$ and $\delta \leqq d(\beta)<\kappa$. This implies $r(\beta)=\kappa$. In addition, by choice of $\delta$, there exists $\gamma \in I$ with $d(\gamma)=\delta<\kappa$ (in this case) and so $r(\gamma)=\kappa$; that is, we can assume without loss of generality that $d(\alpha)=\delta \leqq d(\beta)=\varepsilon$, say. Given all this, we now restrict $\alpha, \beta$ (as in the proof of Theorem 1) to $Y=E(\alpha) \cup E(\beta)$ and obtain $\alpha_{1}, \beta_{1} \in \mathscr{T}_{Y}$ with the same shift, defect and collapse as $\alpha, \beta$ respectively. However, $|Y|=\varepsilon$ and so, from our very first case, $\beta_{1}=\lambda_{1} \alpha_{1} \mu_{1}$ for some $\lambda_{1}, \mu_{1} \in \mathscr{T}_{Y}$ where both $\lambda_{1}$ and $\mu_{1}$ have equal infinite shift, defect and collapse. By extending this equation to the whole of $X$ in an obvious way, we have $\beta=\lambda \alpha \mu$ for some $\lambda, \mu \in H$ and so $\beta \in I$.

Finally, observe that we have indirectly proved

$$
H\left(\delta_{1}, \xi_{1}\right) \subseteq H\left(\delta_{2}, \xi_{2}\right) \text { if and only if } \delta_{1} \geqq \delta_{2} \text { and } \xi_{1} \leqq \xi_{2} \text {, }
$$

and $H\left(d(\alpha), r(\alpha)^{\prime}\right)=H^{1} \alpha H^{1}$ for each $\alpha \in H$.

As noted in ([1], Vol. 2, p. 227, Exercise 3), each $I_{\xi^{\prime}} / I_{\xi}$ is a 0-bisimple semigroup for $\aleph_{0} \leqq \xi \leqq \kappa$. Hence, since $I_{\xi} / I_{\xi}$ contains non-zero idempotents, it is also regular (by [1], Vol. 1, Theorem 2.11). We shall consider the Rees factor semigroups corresponding to the ideals in $\left(^{*}\right)$ after we have determined Green's $\mathscr{D}$ and $\mathscr{J}$ relations on $H$ in Section 3. 
At this point we simply remark that $H(\kappa, \xi)=I_{\xi}$ for each $\xi$ satisfying $1<\xi \leqq \kappa$. For. if $\alpha \in I_{\xi}$ and $1<\xi \leqq \kappa$ then $d(\alpha)=\kappa$ and, since $D(\alpha) \subseteq S(\alpha)$, we also have $s(\alpha)=\kappa$. But $\alpha$ can be written as

$$
\alpha=\left(\begin{array}{ccc}
A_{m} & a_{n} & a_{p} \\
x_{m} & x_{n} & a_{p}
\end{array}\right)
$$

where $C(\alpha)=\cup\left\{A_{m}: m \in M\right\}, a_{n} \neq x_{n}$ for all $n \in N$ and $\left\{a_{p}\right\}=X \backslash[C(\alpha) \cup S(\alpha)]$. Since $|M \cup N|<\kappa$ and $s(\alpha)=\kappa$, we must have $\left|C(\alpha) \backslash\left\{x_{n}\right\}\right|=\kappa$ and so $\alpha \in H(\kappa, \xi)$.

On the other hand, $H\left(\kappa, \kappa^{\prime}\right)$ is a proper subset of $I_{\kappa^{\prime}}$. For, if $\xi<\kappa$ we can partition $X$ into sets $A$ and $B_{1}, B_{2}, B_{3}, \ldots$ where $|A|=\xi$ and $\left|B_{i}\right|=\kappa$ for each $i \geqq 1$, choose bijections $\theta_{i}: B_{i} \rightarrow B_{i+1}$ and $a \in A$, and then define $\alpha \in \mathscr{T}_{X}$ by

$$
\begin{aligned}
& x \alpha=a \quad \text { if } \quad x \in A, \\
& =x \theta_{i} \quad \text { if } \quad x \in B_{i} .
\end{aligned}
$$

Then $D(\alpha)=B_{1}, S(\alpha)=\cup\left\{B_{i}: i \geqq 1\right\}$ and $C(\alpha)=A$; that is $\alpha \notin H\left(\kappa, \kappa^{\prime}\right)$.

Theorem 4. The ideals of $\mathscr{E}_{X}$ are precisely the ideals of $H$ together with the sets $V_{n} \cup H$ for $n \geqq 1$.

Proof. By Lemma 1 and Theorem 3 the ideals of $H$, as well as the sets $V_{n} \cup H$, are all ideals of $\mathscr{E}_{X}$. Conversely, suppose $I$ is an ideal of $\mathscr{E}_{X}$; the desired result follows immediately from Theorem 1 since if $I \cap H$ is a proper subset of $H$ we can use Lemma 1 to obtain a contradiction.

\section{Green's Relations}

For convenience we start this section by re-stating certain information from ([1], Vol. 1 , pp. 52-53).

\section{Lemma 3. If $\alpha, \beta \in \mathscr{T}_{X}$ then}

(a) $\beta=\lambda \alpha$ for some $\lambda \in \mathscr{T}_{X}$ if and only if $X \beta \subseteq X \alpha$,

(b) $\beta=\alpha \mu$ for some $\mu \in \mathscr{T}_{X}$ if and only if $\alpha \circ \alpha^{-1} \subseteq \beta \circ \beta^{-1}$,

(c) $\beta=\lambda \alpha \mu$ for some $\lambda, \mu \in \mathscr{T}_{X}$ if and only if $r(\alpha) \leqq r(\beta)$,

(d) $\mathscr{D}=\mathscr{J}$.

Comparable statements can be made for $\mathscr{P}_{X}$ and the symmetric inverse semigroup $\mathscr{I}_{X}$ on $X$ (see [5] for a brief summary of this idea and its extension to a categorical setting). Our task in this section is to show that statements analogous to $(a),(b)$ and $(d)$ hold for both $V$ and $H$, but that something different occurs for $(c)$.

Theorem 5. If $\alpha, \beta \in V$ then

(a) $\beta=\lambda \alpha$ for some $\lambda \in V$ if and only if $X \beta \subseteq X \alpha$, 
(b) $\beta=\alpha \mu$ for some $\mu \in V$ if and only if $\alpha \circ \alpha^{-1} \subseteq \beta \circ \beta^{-1}$,

(c) $\beta=\lambda \alpha \mu$ for some $\lambda, \mu \in V$ if and only if $d(\beta) \geqq d(\alpha)$,

(d) $\mathscr{D}=\mathscr{J}$.

Proof. Suppose $X \alpha \subseteq X \beta$ where $X \beta$ is a proper subset of $X$. Put $Y=E(\alpha) \cup E(\beta)$ and restrict $\alpha, \beta$ to $Y$ to produce $\alpha_{1}, \beta_{1} \in \mathscr{T}_{Y}$ where $Y \beta_{1}$ is a proper subset of $Y$. Now $Y$ is finite and $Y \beta_{1} \subseteq Y \alpha_{1}$ : since if $y \in Y$ and $y \beta=x \alpha$ for $x \in X$ then $x \alpha \neq x$ (and so $x \in Y$ ) or $x \alpha=x$ (and so $x=y \beta \in Y$ ). By Lemma 3(a), $\beta_{1}=\lambda_{1} \alpha_{1}$ for some $\lambda_{1} \in \mathscr{T}_{Y}$ which moreover can be chosen with $d\left(\lambda_{1}\right) \neq 0$ (since $d\left(\beta_{1}\right) \neq 0$ ). Then $\beta=\lambda \alpha$ where $\lambda \in V$ and part (a) follows; a similar argument establishes part (b).

Suppose $\beta=\lambda \alpha \mu$ where $d(\alpha)=n$. By Theorem $1, V^{1} \alpha V^{1}=V_{n}$ and so $d(\beta) \geqq n$. Conversely, suppose $d(\beta) \geqq d(\alpha)$. Then, using our customary notation, $r\left(\beta_{1}\right) \leqq r\left(\alpha_{1}\right)$ and Lemma 3(c) implies $\beta_{1}=\lambda_{1} \alpha_{1} \mu_{1}$ for some $\lambda_{1}, \mu_{1} \in \mathscr{T}_{Y}$. In fact, since $Y$ is finite and $d\left(\beta_{1}\right) \neq 0$, both $\lambda_{1}$ and $\mu_{1}$ can be chosen with non-zero defect; hence we have $\beta=\lambda \alpha \mu$ with $\lambda, \mu \in V$, as required.

Finally, if $V^{1} \alpha V^{1}=V^{1} \beta V^{1}$ then $d(\alpha)=d(\beta) \neq 0$ and so $r\left(\alpha_{1}\right)=r\left(\beta_{1}\right) \neq|Y|$. By Lemma 3(d), this implies $\alpha_{1} \mathscr{L}_{\gamma_{1}} \mathscr{R} \beta_{1}$ for some $\gamma_{1} \in \mathscr{T}_{Y}$ which can in fact be chosen with non-zero defect. Hence, $\alpha \mathscr{L} \gamma \mathscr{R} \beta$ for some $\gamma \in V$ and the proof is complete.

The proof of the corresponding result for $H$ is much longer since our technique of restricting $\alpha, \beta \in \mathscr{T}_{X}$ to $Y=E(\alpha) \cup E(\beta)$ does not seem to help matters.

Theorem 6. If $\alpha, \beta \in H$ then

(a) $\beta=\lambda \alpha$ for some $\lambda \in H$ if and only if $X \beta \subseteq X \alpha$,

(b) $\beta=\alpha \mu$ for some $\mu \in H$ if and only if $\alpha \circ \alpha^{-1} \subseteq \beta \circ \beta^{-1}$,

(c) $\beta=\lambda \alpha \mu$ for some $\lambda, \mu \in H$ if and only if $r(\beta) \leqq r(\alpha)$ and $d(\beta) \geqq d(\alpha)$,

(d) $\mathscr{D}=\mathscr{J}$.

Proof. Suppose $X \beta \subseteq X \alpha$ and put $Z=X \backslash[C(\beta) \cup S(\beta)]$. Then $d(\beta) \geqq d(\alpha)$ and $|Z \cap S(\alpha)| \leqq s(\alpha) \leqq s(\beta)$. Now write

$$
\beta=\left(\begin{array}{llll}
B_{p} & b_{q} & b_{m} & b_{n} \\
x_{p} & x_{q} & b_{m} & b_{n}
\end{array}\right)
$$

where $C(\beta)=\cup\left\{B_{p}: p \in P\right\}, b_{q} \neq x_{q}$ for all $q \in Q,\left\{b_{m}\right\}=Z \cap S(\alpha)$, and $Z=\left\{b_{m}\right\} \dot{\cup}\left\{b_{n}\right\}$. Then $b_{n} \alpha=b_{n}$ for all $n \in N$ and we can write

$$
\alpha=\left(\begin{array}{lllll}
A_{p} & A_{q} & A_{m} & A_{n} & A_{s} \\
x_{p} & x_{q} & b_{m} & b_{n} & x_{s}
\end{array}\right)
$$

where $b_{n} \in A_{n}$ for each $n \in N$ and $\left\{x_{s}\right\}=X \alpha \backslash X \beta$ (if non-empty). We now choose a partial cross-section $\left\{a_{p}\right\} \dot{\cup}\left\{a_{q}\right\} \dot{\cup}\left\{a_{m}\right\} \dot{\cup}\left\{b_{n}\right\}$ of $X / \alpha \circ \alpha^{-1}$ and put

$$
\lambda=\left(\begin{array}{llll}
B_{p} & b_{q} & b_{m} & b_{n} \\
a_{p} & a_{q} & a_{m} & b_{n}
\end{array}\right) .
$$


Note that since $|M| \leqq s(\beta)$, we have $c(\lambda)=s(\lambda)=s(\beta)$. In addition, we have

$$
D(\lambda) \subseteq\left[C(\beta) \cup S(\beta) \cup\left\{b_{m}\right\}\right] \backslash\left[\left\{a_{p}\right\} \cup\left\{a_{q}\right\} \cup\left\{a_{m}\right\}\right]
$$

and so $d(\lambda) \leqq s(\beta)$. If $d(\alpha)<d(\beta)$ then $|S|=d(\beta)$ and so, since $\cup\left\{A_{s}: s \in S\right\} \subseteq D(\lambda)$, we have $d(\lambda)=s(\beta)$. Hence, we may suppose $d(\alpha)=d(\beta)=\varepsilon$ say. Now write $C(\alpha)=\cup\left\{A_{t}: t \in T\right\}$ and note that, when selecting the partial cross-section of $X / \alpha \circ \alpha^{-1}$ to form $\lambda$, we choose at most one element from each $A_{t}$. Consider the worst case and suppose we have in fact chosen some $a_{t} \in A_{t}$ for each $t \in T$. Since each $A_{t}$ contains at least 2 elements, we have $\left|C(\alpha) \backslash\left\{a_{t}\right\}\right|=\varepsilon$. However, $C(\alpha) \backslash\left\{a_{t}\right\} \subseteq D(\lambda)$ and so $d(\lambda)=\varepsilon$. That is, $\lambda \in H$ and $\beta=\lambda \alpha$.

For part (b), we now suppose $\alpha \circ \alpha^{-1} \subseteq \beta \circ \beta^{-1}$ and write

$$
\begin{aligned}
& \beta=\left(\begin{array}{lllll}
B_{m} & B_{p} & x_{i} & w_{r} & w_{s} \\
b_{m} & b_{p} & y_{i} & w_{r} & w_{s}
\end{array}\right) \\
& \alpha=\left(\begin{array}{lllll}
B_{m n} & B_{p} & x_{i} & w_{r} & w_{s} \\
c_{m n} & c_{p} & z_{i} & v_{r} & w_{s}
\end{array}\right)
\end{aligned}
$$

where the sets $B_{m}$ and $B_{p}$ contain at least 2 elements, $B_{m}=\cup\left\{B_{m n}: n \in N_{m}\right\}$ for some index set $N_{m}$, and $x_{i} \neq y_{i}, w_{r} \neq v_{r}$ (note that possibly $x_{i}=z_{i}$ for some $i$, and also some $B_{m n}$ may consist of a single element). The above display is possible since each $\beta \circ \beta^{-1}$-class is the union of one or more $\alpha \circ \alpha^{-1}$-classes; the sets $N_{m}$ are therefore chosen to satisfy $2 \leqq\left|N_{m}\right| \leqq c(\beta)$. Put $C_{m}=\left\{c_{m n}: n \in N_{m}\right\}$ and $D=D(\alpha)$, choose $d \in D$ and let

$$
\mu=\left(\begin{array}{llllll}
C_{m} & c_{p} & z_{i} & v_{r} & w_{s} & D \\
b_{m} & b_{p} & y_{i} & w_{r} & w_{s} & d
\end{array}\right)
$$

Then $\beta=\alpha \mu$ and $D(\mu)=D(\beta) \backslash d$ : that is, $d(\mu)=d(\beta)$. In addition, $C(\mu)=\left(\cup C_{m}\right) \cup D$ and

$$
S(\mu) \subseteq\left(\cup C_{m}\right) \cup\left\{c_{p}\right\} \cup\left\{z_{i}\right\} \cup\left\{v_{r}\right\} \cup D .
$$

However, $C(\alpha) \subseteq C(\beta)$ and so $d(\alpha) \leqq d(\beta)$; also, $|M \cup P| \leqq c(\beta),|I| \leqq s(\beta),|R| \leqq s(\alpha)$ and $\left|C_{m}\right| \leqq c(\beta)$ for each $m$. Hence both $c(\mu)$ and $s(\mu)$ are at most $d(\beta)$. In fact, it is clear from the very definition of $\mu$ that $c(\mu)=s(\mu)=d(\beta)$ when $d(\alpha)=d(\beta)$. So, suppose $d(\alpha)<d(\beta)$. This implies $\left|\cup B_{m}\right|=c(\beta)$ since $\left|\cup B_{p}\right| \leqq c(\alpha)$; also $\left|\left(\cup B_{m}\right) \cap S(\alpha)\right|<c(\beta)$. Hence, $\left|\left(\cup B_{m}\right) \cap F(\alpha)\right|=c(\beta)$ where $F(\alpha)=X \backslash S(\alpha)$. But $\cup B_{m}=\bigcup_{m} \bigcup_{n} B_{m n}$ and so in this case there are $c(\beta)$ elements in $\cup C_{m}$ that are fixed by $\alpha$. Consequently, $\left|\cup C_{m}\right|=c(\beta)$ and so $c(\mu)=c(\beta)$. Moreover, each $C_{m}$ contains at least 2 elements and $|M| \leqq c(\beta)$. So, $\left|\cup\left(C_{m} \backslash b_{m}\right)\right|=c(\beta)$ and therefore $s(\mu) \geqq c(\beta)$. That is, $\mu \in H$ as required.

To prove parts (c) and (d), we first show that $\alpha \mathscr{D} \beta$ in $H$ if and only if $r(\alpha)=r(\beta)$ and $d(\alpha)=d(\beta)$. Suppose $\alpha \mathscr{L} \gamma \mathscr{R} \beta$ for some $\gamma \in H$. By parts (a) and (b), $X \alpha=X \gamma$ and $\gamma \circ \gamma^{-1}=$ $\beta \circ \beta^{-1}$. Hence, $r(\alpha)=r(\beta)$ and $d(\alpha)=d(\gamma)=c(\gamma)=c(\beta)=d(\beta)$. For the converse we assume $r(\alpha)=r(\beta)$ and $d(\alpha)=d(\beta)$, and consider two cases. If $d(\alpha)=\kappa$ we choose any $\gamma \in \mathscr{T}_{X}$ with $X \alpha=X \gamma$ and $\gamma \circ \gamma^{-1}=\beta \circ \beta^{-1}$ (such $\gamma^{\text {'s exist since } r(\alpha)=r(\beta) \text { ). Then } d(\gamma)=d(\alpha)=\kappa}$ (and so $s(\gamma)=\kappa$ since $D(\gamma) \subseteq S(\gamma)$ ) and $c(\gamma)=c(\beta)=\kappa$; that is, $\gamma \in H$ and we are finished. 
If on the other hand $d(\alpha)=\delta<\kappa$ then $r(\alpha)=\kappa$ and our task of finding a suitable $\gamma$ is much harder. However, before accomplishing this we note in passing that this case cannot be reduced to the one already considered by restricting $\alpha, \beta$ to $Y=E(\alpha) \cup E(\beta)$. For, we might now have

$$
\alpha=\left(\begin{array}{cc}
U \cup V & c_{n} \\
x & c_{n}
\end{array}\right) \quad \beta=\left(\begin{array}{ccc}
U & V & c_{n} \\
y & z & c_{n}
\end{array}\right)
$$

where $U, V$ and $\left\{c_{n}\right\}$ partition $X,|U|=|V|=\delta<\kappa$, and $x, y, z$ are distinct elements of $U \cup V$; if this were so then $Y=U \cup V$ and $d\left(\alpha_{1}\right)=d\left(\beta_{1}\right)=\delta$ but $r\left(\alpha_{1}\right) \neq r\left(\beta_{1}\right)$.

Now let $A=X \alpha \backslash[C(\beta) \cup S(\beta)]$ and note that $|A|=\kappa$ and $a \beta=a$ for all $a \in A$. Put

$$
\begin{aligned}
& B=X \alpha \cap[C(\beta) \cup S(\beta)] \\
& C=D(\alpha) \cup C(\beta) \cup S(\beta) \\
& \varepsilon=\max \left(|B|,|C \beta|, \aleph_{0}\right)
\end{aligned}
$$

and note that $\aleph_{0} \leqq \varepsilon \leqq \delta<\kappa$ since $|C|=\delta$. Choose a subset $D$ of $A$ with $|D|=\varepsilon$ and let $E=C \cup D, F=B \cup D$. Now $|E \beta|=|C \beta|+|D \beta|=\varepsilon$ (since $D \beta=D$ ) and $|F|=\varepsilon$. Let $\theta: E \beta \rightarrow F$ be any bijection and define $\gamma \in \mathscr{T}_{X}$ by

$$
\begin{aligned}
x y & =x \quad \text { if } x \in A \backslash D \\
& =x \beta \theta \text { if } x \in E .
\end{aligned}
$$

The domain of $\gamma$ is $X$ since $E \cup(A \backslash D)$ contains $C \cup A$ which equals $X \alpha \cup D(\alpha)$. Moreover, if $x y=y \gamma$ then either (1) $x=y \in A \backslash D$, or (2) $x \in A \backslash D, y \in E$ and $x=y \beta \theta$, or (3) $x, y \in E$ and $x \beta \theta=y \beta \theta$ (we omit the dual of (2)). If (1) occurs then $x \beta=y \beta$; if (2) occurs then $x \in F$ and so $x \in B$ (since $x \notin D$ ), contradicting the assumption that $x \in A$; and if (3) occurs then $x \beta=y \beta$ since $\theta$ is one-to-one. That is, $\gamma \circ \gamma^{-1} \subseteq \beta \circ \beta^{-1}$. On the other hand, if $x \beta=y \beta$ then either $x=y$ (and so $x y=y \gamma$ ) or $x \neq y$ (in which case $x, y \in C(\beta) \subseteq E$ and so $x \gamma=x \beta \theta=y \beta \theta=y \gamma)$. Hence, $\gamma \circ \gamma^{-1}=\beta \circ \beta^{-1}$. In addition,

$$
X \gamma=[E \cup(A \backslash D)] \gamma=F \cup(A \backslash D)=A \cup B=X \alpha .
$$

Thus, $c(\gamma)=c(\beta)=d(\alpha)=d(\gamma)$. Clearly, $S(\gamma) \subseteq E$ and $|E|=\delta$. But $D(\gamma) \subseteq S(\gamma)$ and $d(\gamma)=\delta$; thus, $s(\gamma)=\delta$ and we have found some $\gamma \in H$ such that $\alpha \mathscr{L} \gamma \mathscr{R} \beta$.

Having characterised Green's $\mathscr{D}$ relation on $H$, we now consider part (c) and suppose $\beta=\lambda \alpha \mu$. Then $r(\beta) \leqq r(\alpha)$ and $d(\beta) \geqq d(\mu)$. Hence, if $d(\beta)<d(\alpha)$ then $d(\alpha \mu)=d(\alpha)$ (by Lemma 1) as well as $d(\beta) \geqq d(\alpha \mu)$, a contradiction. Therefore $d(\beta) \geqq d(\alpha)$. For the converse suppose $\alpha, \beta \in H, r(\beta) \leqq r(\alpha)$ and $d(\beta) \geqq d(\alpha)$. This means $\beta \in H\left(d(\alpha), r(\alpha)^{\prime}\right)$ which by Theorem 3 equals $H^{1} \alpha H^{1}$, and so part (c) is proved. Finally, $\alpha \mathscr{J} \beta$ implies $H^{1} \alpha H^{1}=H^{1} \beta H^{1}$ and this in turn implies $d(\alpha)=d(\beta)$ and $r(\alpha)=r(\beta)$; from the foregoing, we deduce $\alpha \mathscr{D} \beta$, and of course $\mathscr{D} \subseteq \mathscr{J}$ always.

It may be worthwhile illustrating the choice of $\gamma$ for the $\alpha, \beta$ displayed in (**) above. Using the notation introduced in the second last paragraph of the proof, we have 
$A=\left\{c_{n}\right\}, B=\{x\}, C=U \cup V$ and $\varepsilon=\aleph_{0}$. Then we in effect "blow-up" $r\left(\alpha_{1}\right)$ and $r\left(\beta_{1}\right)$ until they are equal by suitably enlarging the domain of $\alpha_{1}$ and $\beta_{1}$. That is, we choose $D=\left\{c_{m}\right\}$ in $A$ with $|M|=\varepsilon=\aleph_{0}$ and note that $E \beta=\{y, x\} \cup\left\{c_{m}\right\}$ has the same cardinal as $F=\{x\} \cup\left\{c_{m}\right\}$. If $\left\{c_{p}\right\}=A \backslash D$ then $\gamma$ is the map

$$
\left(\begin{array}{cccc}
U & V & c_{m} & c_{p} \\
y \theta & z \theta & c_{m} \theta & c_{p}
\end{array}\right)
$$

where $\theta$ is any bijection between $E \beta$ and $F$.

Before proceeding we note that the significance of Theorem 6 (especially part (d)) lies in the fact that it gives some hope of determining the congruences on $H$ in a manner akin to that developed by Clifford and Preston for $\mathscr{T}_{X}$ in ([1], Vol. 2, Section 10.8); we shall explore this possibility in a subsequent paper.

We now consider the Rees quotient semigroup $H\left(\delta, \kappa^{\prime}\right) / H\left(\delta^{\prime}, \kappa^{\prime}\right)$ for $\aleph_{0} \leqq \delta<\kappa$. Clearly, each non-zero element of this semigroup has defect $\delta$ and rank $\kappa$. Moreover, a close perusal of the proof of Theorem 6 shows that if $d(\alpha)=d(\beta)$ and $r(\alpha)=r(\beta)$ for $\alpha, \beta \in H$ then there exists $\gamma \in H$ such that $\alpha \mathscr{L} \gamma \mathscr{R} \beta$ and $d(\gamma)=d(\alpha)$. In addition, if $\alpha \mathscr{L} \gamma$ in $H$ then there exist $\lambda_{1}, \lambda_{2} \in H$ with $\alpha=\lambda_{1} \gamma, \gamma=\lambda_{2} \alpha$ and $d\left(\lambda_{1}\right)=d(\alpha), d\left(\lambda_{2}\right)=d(\gamma)$; likewise, if $\gamma \mathscr{R} \beta$ in $H$ then there exist $\mu_{1}, \mu_{2} \in H$ with $\gamma=\beta \mu_{1}, \beta=\gamma \mu_{2}$ and $d\left(\mu_{1}\right)=d(\gamma), d\left(\mu_{2}\right)=d(\beta)$. In other words, each $H\left(\delta, \kappa^{\prime}\right) / H\left(\delta^{\prime}, \kappa^{\prime}\right)$ is 0 -bisimple when $\delta<\kappa$. Each such semigroup is also regular since it contains non-zero idempotents and ([1], Vol. 1, Theorem 2.11) can be applied. However, none of them is completely 0 -simple since they always contain nonzero non-primitive idempotents; for example, if

$$
\alpha=\left(\begin{array}{lll}
A & b_{m} & b_{n} \\
a & b_{m} & b_{n}
\end{array}\right) \quad \beta=\left(\begin{array}{cc}
A \cup\left\{b_{m}\right\} & b_{n} \\
a & b_{n}
\end{array}\right)
$$

where $|A|=\left|\left\{b_{m}\right\}\right|=\delta$ and $a \in A$, then $\alpha, \beta$ are distinct idempotents satisfying $\alpha \beta=\beta \alpha=\beta$.

Unfortunately we cannot decide whether these 0-bisimple quotients in the "top half" of $\left(^{*}\right)$ are isomorphic to any of the quotients in the "bottom half" of $\left(^{*}\right)$. For, an argument similar to that applied to $V_{n} / V_{n+1}$ in Section 2 can be used to show that for $\delta<\kappa, H\left(\delta, \kappa^{\prime}\right) / H\left(\delta^{\prime}, \kappa^{\prime}\right)$ has cardinal $\kappa^{\delta}$ (this is because the set of all subsets of $X$ with cardinal $\delta$ has cardinal $\kappa^{\delta}$ : see [12], Exercise 22.25). It can also be readily shown that the non-zero group $\mathscr{H}$-classes of $H\left(\delta, \kappa^{\prime}\right) / H\left(\delta^{\prime}, \kappa^{\prime}\right)$ are all isomorphic to the group $\mathscr{G}\left(\kappa, \delta^{\prime}\right)$ of all permutations of $\kappa$ letters with shift at most $\delta$. On the other hand, for $\xi \leqq \kappa, I_{\xi} / I_{\xi}$ has cardinal $2^{\kappa}$ and its non-zero group $\mathscr{H}$-classes are all isomorphic to $\mathscr{G}_{\xi}$. However, without $G C H$, we may have $2^{\delta}=2^{\xi}$ even though $\delta \neq \xi([13]$, pp. 119 and 130).

Our final result in this section in effect determines Green's relations on $\mathscr{E}_{X}$.

Theorem 7. If $\alpha, \beta \in \mathscr{E}_{X}$ and are related under one of Green's relations on $\mathscr{E}_{X}$ then $\alpha, \beta \in V$ or $\alpha, \beta \in H$.

Proof. Suppose $\alpha \in V, \beta \in H$ and $\alpha=\lambda \beta$ for some $\lambda \in \mathscr{E}_{X}$. Then, by Lemma 1, $s(\lambda) \geqq s(\beta)$ and this means $\lambda \in H$ which in turn implies $\alpha \in H$, a contradiction. A similar argument can be applied if $\alpha=\beta \mu$ or if $\alpha=\lambda \beta \mu$ for some $\lambda, \mu \in \mathscr{E}_{X}$. 


\section{Partial Transformations}

In this section we consider the way in which the results of Sections 2 and 3 can be extended to the semigroup $\mathscr{E}_{X}^{*}$ that is generated by all the idempotent partial transformations of $X$ different from the identity (it is clear that the identity cannot be written as a product of idempotents in $\mathscr{P}_{X}$ different from the identity).

For finite $X$, the elements of $\mathscr{E}_{X}^{*}$ were characterised by Evseev and Podran [3] (and independently by Sullivan [15]). As one might expect (by analogy with $\mathscr{T}_{X}$ ), if $|X|=n$ then $\mathscr{E}_{X}^{*}=\left\{\alpha \in \mathscr{P}_{X}: r(\alpha)<n\right\}$ and moreover each $\alpha \in \mathscr{E}_{X}^{*}$ can in fact be written as a product of idempotents in $\mathscr{P}_{X}$ with defect 1 (note that such idempotents can equal $l_{Y}$ for some $Y \subseteq X$ with $|Y|=n-1)$. Given this, it is easy to see what the ideals and Green's relations on $\mathscr{E}_{X}^{*}$ must be when $X$ is finite.

Hence we again assume throughout this section that $|X|=\kappa \geqq \aleph_{0}$. It seems from a review that Evseev and Podran [4] have also investigated $\mathscr{E}_{X}^{*}$ in this case. Since we need a straight forward characterisation of the elements of $\mathscr{E}_{X}^{*}$ in order to describe the ideals of $\mathscr{E}_{X}^{*}$, we now present such a characterisation and for completeness we include a short proof based on Howie's Theorem.

However, before proceeding to do this we recall Lyapin's method of representing $\mathscr{P}_{X}$ as a semigroup of total transformations: namely, let $0 \notin X$, put $Y=X \cup 0$ and

$$
F_{0}=\left\{\beta \in \mathscr{T}_{Y}: 0 \beta=0\right\} \text {, }
$$

and define $\theta: \mathscr{P}_{X} \rightarrow F_{0}, \alpha \rightarrow \alpha \theta$, where $x(\alpha \theta)=x \alpha$ if $x \in \operatorname{dom} \alpha$ and $x(\alpha \theta)=0$ otherwise. Clearly $\theta$ is an isomorphism. We extend the notions of defect, collapse and shift of $\alpha \in \mathscr{T}_{X}$ to elements of $\mathscr{P}_{X}$ as follows: for each $\alpha \in \mathscr{P}_{X}$, let

$$
\begin{array}{lll}
D^{*}(\alpha)=X \backslash X \alpha & \text { and } & d^{*}(\alpha)=\left|D^{*}(\alpha)\right| \\
C^{*}(\alpha)=C(\alpha) \cup(X \backslash \operatorname{dom} \alpha) & \text { and } & c^{*}(\alpha)=\left|C^{*}(\alpha)\right| \\
S^{*}(\alpha)=\{x \in \operatorname{dom} \alpha: x \alpha \neq x\} \cup(X \backslash \operatorname{dom} \alpha) & \text { and } & s^{*}(\alpha)=\left|S^{*}(\alpha)\right|
\end{array}
$$

Theorem 8. An element $\alpha$ of $\mathscr{P}_{X}$ can be written as a product of idempotents in $\mathscr{P}_{X}$ different from the identity if and only if either $s^{*}(\alpha)<\aleph_{0}$ and $d^{*}(\alpha) \neq 0$ or $s^{*}(\alpha)=c^{*}(\alpha)=$ $d^{*}(\alpha) \geqq \aleph_{0}$.

Proof. Suppose $\alpha \in \mathscr{E}_{X}^{*}$ and $\beta=\alpha \theta$. Then $\beta \in \mathscr{E}_{Y}$ and so, by Howie's Theorem, either $s(\beta)<\aleph_{0}$ and $d(\beta) \neq 0$ or $s(\beta)=c(\beta)=d(\beta) \geqq \aleph_{0}$. Since $Y \backslash Y \beta=X \backslash X \alpha, C(\beta)=C^{*}(\alpha)$ and $S(\beta)=S^{*}(\alpha)$, this produces the desired result. Now suppose $\alpha \in \mathscr{P}_{X}$ and $\alpha$ satisfies $s^{*}(\alpha)=$ $c^{*}(\alpha)=d^{*}(\alpha) \geqq \aleph_{0}$. Then $\beta=\alpha \theta \in F_{0}$ and $\beta$ satisfies the corresponding condition in Howie's Theorem. Suppose

$$
\beta=\left(\begin{array}{llll}
B_{i} & C & y_{j} & a_{n} \\
x_{i} & 0 & z_{j} & a_{n}
\end{array}\right)
$$


where each $B_{i}$ contains at least 2 elements and $y_{j} \neq z_{j}$ for each $j$. Choose $b_{i} \in B_{i}$ and let

$$
\lambda=\left(\begin{array}{llll}
B_{i} & C & y_{j} & a_{n} \\
b_{i} & 0 & y_{j} & a_{n}
\end{array}\right) \quad \mu=\left(\begin{array}{ccccc}
b_{i} & 0 & y_{j} & a_{n} & D \\
x_{i} & 0 & z_{j} & a_{n} & d
\end{array}\right)
$$

where $D=(C \backslash 0) \cup\left(C(\beta) \backslash\left\{b_{i}\right\}\right)$ and $d \in D$. Then $\beta=\lambda \mu$ where $\lambda^{2}=\lambda \in F_{0}$. Now since $c(\beta)$ equals either $\left|\cup B_{i}\right|$ or $|C|$, we have $|D|=c(\beta)$. Thus, since $|I| \leqq c(\beta)$ and $|J| \leqq s(\beta)$, we have $c(\mu)=s(\mu)=c(\beta)$. Moreover, $D(\mu)=D(\beta) \backslash d$ and so $d(\mu)=s(\beta)$. Therefore, by Howie's Theorem, $\mu \mid X$ is a product of idempotents in $\mathscr{T}_{X}$, each of which can be extended in an obvious way to an idempotent in $F_{0}$. By applying the isomorphism $\theta^{-1}$, we obtain a product of idempotents in $\mathscr{P}_{X}$ that equals $\alpha$.

Since the proof for the case when $s^{*}(\alpha)<\aleph_{0}$ and $d^{*}(\alpha) \neq 0$ can be carried through in an entirely similar manner, we omit the details. However we note that in this case $\beta$ cannot look like

$$
\left(\begin{array}{lll}
0 & y_{j} & a_{n} \\
0 & z_{j} & a_{n}
\end{array}\right)
$$

where $|J|<\aleph_{0}$ for this would mean $\beta$ is a permutation, contradicting $d(\beta) \neq 0$. In other words, either $|C| \geqq 2$ or $I \neq \square$ : this fact can be used to ensure that defects are non-zero.

We can now write $\mathscr{E}_{X}^{*}=V^{*} \cup H^{*}$ where

$$
\begin{gathered}
V^{*}=\left\{\alpha \in \mathscr{P}_{X}: s^{*}(\alpha)<\aleph_{0} \text { and } d^{*}(\alpha) \neq 0\right\} \\
H^{*}=\left\{\alpha \in \mathscr{P}_{X}: s^{*}(\alpha)=d^{*}(\alpha)=c^{*}(\alpha) \geqq \aleph_{0}\right\}
\end{gathered}
$$

and $\square \in H^{*}$. Using the results of Section 2 and the isomorphism $\theta$, it is a simple matter to check that the ideals of $V^{*}$ take the form

$$
V_{n}^{*}=\left\{\alpha \in V^{*}: d^{*}(\alpha) \geqq n\right\}
$$

where $n \geqq 1$, and the ideals of $H^{*}$ equal

$$
H^{*}(\delta, \xi)=\left\{\alpha \in H^{*}: d^{*}(\alpha) \geqq \delta \quad \text { and } \quad r(\alpha)<\xi\right\}
$$

for some $\delta, \xi$ satisfying $\aleph_{0} \leqq \delta \leqq \kappa$ and $1 \leqq \xi \leqq \kappa^{\prime}$. In addition, Green's relations on $V^{*}$ and on $H^{*}$ are precisely what one would expect given the results of Section 3.

\section{REFERENCES}

1. A. H. Clifford and G. B. Preston, The Algebraic Theory of Semigroups (Math. Surveys, no. 7, Amer. Math. Soc., Providence, RI, Vol. 1, 1961; Vol. 2, 1967).

2. J. A. ERDos, On products of idempotent matrices, Glasgow Math. J. 8 (1967), 118-122.

3. A. E. Evseev and N. E. Podran, Semigroups of transformations generated by idempotents with given projection characteristics, Isv. Vyss. Ucebn. Zaved. Mat. 12 (103), 1970, 30-36. 
4. A. E. Evseev and N. E. Podran, Semigroups of transformations generated by idempotents with given defect, Izv. Vyss. Ucebn. Zaved. Mat. 2 (117) 1972, 44-50.

5. D. G. Fitzgerald and G. B. Preston, Divisibility of binary relations, Bull. Austral. Math. Soc. 5 (1971), 75-86.

6. J. M. HowIE, The subsemigroup generated by the idempotents of a full transformation semigroup, J. London Math. Soc. 41 (1966), 707-716.

7. J. M. HowIE, Some subsemigroups of infinite full transformation semigroups, Proc. Royal Soc. Edin. 88A (1981), 159-167.

8. JiN BaI Kim, Idempotents in symmetric semigroups, J. Combin. Theory 13 (1972), 155-161.

9. E. S. LJAPIN, Semigroups, 3 ed. (Vol. 3, Translations Math. Monographs, Amer. Math. Soc., Providence, RI, 1974).

10. K. D. Magill, JR., The semigroup of endomorphisms of a Boolean ring, J. Austral. Math. Soc. (Series A) 11 (1970), 411-416.

11. K. D. MAGILL, JR., $K$-structure spaces of semigroups generated by idempotents, $J$. London Math. Soc. 3 (1971), 321-325.

12. J. D. Monk, Introduction to Set Theory (McGraw-Hill, NY, 1969).

13. J. B. Rosser, Simplified Independence Proofs (Academic, NY, 1969).

14. B. M. Schern, Products of idempotent order-preserving transformations of arbitrary chains, Semigroup Forum 11 (1975/76), 297-309.

15. R. P. Sullivan, $A$ study in the theory of transformation semigroups (Ph.D. thesis, Monash University, 1969).

16. J. S. V. Symons, Normal transformation semigroups, J. Austral. Math. Soc. (Series A) 22 (1976), 385-390.

17. N. N. Vorobev, Defect ideals of associative systems, Leningrad Gos. Univ. Ucen. Zap., Ser. Mat. Nauk 16 (1949), 47-53.

18. N. N. Vorobev, On symmetric associative systems, Leningrad Gos. Ped. Inst. Ucen. Zap. 89 (1953), 161-166.

Mathematics Department

University of Western Australia

Nedlands, 6009

Western Australia 